\title{
机电一体化数控技术在机械制造中的应用探讨
}

\author{
王卫平 \\ 江西特种电机股份有限公司，江西 宜春 336000
}

\begin{abstract}
[摘要]我国信息科技不断发展促使机械制造行业迎来新的发展机遇。信息技术有效提升了机械制造的水平，尤其是自动化技 术的应用, 推动了机械制造产品质量的提升。机电一体化数控技术在机械制造行业中发挥的作用越来越明显, 企业想要实现 持续健康地发展就要积极引入新技术, 科学选择机械制造技术, 把控数控技术的优势和特点, 不断提高一体化数控技术的应 用水平，保证数控技术能够为机械制造创造更大的价值，推动我国自动化机械制造行业的发展，提升我国机械产品制造的质量。 [关键词]机电一体化; 数控技术; 机械制造; 应用
\end{abstract}

DOI: 10.33142/aem.v2i11.3201 中图分类号: TD67 文献标识码：A

\section{Application of Mechatronics Numerical Control Technology in Mechanical Manufacturing}

\author{
WANG Weiping \\ Jiangxi Special Motor Co., Ltd., Yichun, Jiangxi, 336000, China
}

\begin{abstract}
With the continuous development of information technology in China, machinery manufacturing industry ushers in new development opportunities. Information technology effectively improves the level of mechanical manufacturing, especially the application of automation technology, promotes the improvement of mechanical manufacturing product quality. Mechatronics numerical control technology plays an increasingly important role in the mechanical manufacturing industry. If enterprises want to achieve sustainable and healthy development, they should actively introduce new technology, scientifically select mechanical manufacturing technology, control the advantages and characteristics of numerical control technology, and constantly improve the application level of integrated numerical control technology, so as to ensure that numerical control technology can create greater value for mechanical manufacturing and promote the development of our country. With the development of Chinese automatic machinery manufacturing industry, the quality of Chinese mechanical product manufacturing will be improved.
\end{abstract}

Keywords: mechatronics; numerical control technology; mechanical manufacturing; application

\section{1 机电一体化技术概述}

我国信息科技在近些年发展迅速, 信息科技的发展带动了机电一体化技术的进步, 同时相关研究者也开始深入研 究和促进机电一体化技术的整合。作为一门独立的可续, 机电一体化技术从提出至今已经经过了多年发展, 很多高校 都已经专门设置机电一体化技术学科, 加大机电一体化人才的培养, 从而满足社会对机电一体化人才的需求。目前我 国市场仍然缺乏专业的人才, 需要进一步加强机电一体化技术普及和人才培养。具体来讲, 机电一体化技术主要涉及 两方面的内容, 一是相关产品, 二是相关技术。对于相关工作者来讲, 如何高效运用和研究机电一体化是工作重难点, 需要充分考虑机电一体化产品与机电一体化技术之间的密切关系, 避免过度使用创新技术造成两者脱离的问题, 要合 理创新, 保证以实际生产为基础进行合理改进优化。在研究机电一体化技术进程中, 需要加强融合该机属于先进的科 学技术, 从而保证产品质量更高, 进而保证机电一体化实现持续稳定地发展。目前主要从如下几个方面应用机电一体 化技术: 第一, 机械技术是机电一体化技术中最为基础的内容, 良好地融合机电一体化技术和机械技术能够推动我国 机电一体化技术的创新发展, 有助于改进人们对机电一体化的认识, 有助于以机电一体化技术性能、材料为基础进行 进一步改进创新, 创造体积更小、重量更轻、精准度更高的设备, 更好地满足生产需要。在机电一体化技术系统创设 时需要科学地运用机械设备制作工艺和理论知识, 加强对计算机辅助技术的应用, 加强专业计算机系统和人工智能技 术的应用，让先进的信息技术助理机械制造行业的发展。第二，信息计算机技术。该技术主要是存储、收集、交换、 运算、决策、判断相关的信息数据。计算机信息基础处理内容包括计算机技术系统、人工智能技术等。第三，自动控 制技术。当前机电一体化中已经广泛地应用自动控制技术, 想要进一步优化自动控制系统就要积极应用高标准的理论 认真完成现场调试和系统仿真等自动化技术。目前速度控制、方位控制、自动适应控制、自动诊断、自动补偿等都是 
机电一体化控制技术常见的功能。第四，传感技术。传感技术作为整个系统感受组成部分在自动化控制和自动调节方 面发挥着非常重要的作用, 传感技术可以在机械制造中准确地获取系统中的信息内容, 能够将所需要的信息资源十分 快速地获取, 有助于机电一体化技术系统应用水平的高效提升。

\section{2 机电一体化数控技术的实际应用}

\section{1 应用在传感器部分}

传感器时机电一体化数控技术系统中传输信息的主要构件, 传感器复杂向不同的元件传输系统内部的信息, 从而 保证各个元件能够按照收到的指令开始工作。利用传感器不但能够将信息传输的效率提高, 还可以保障信息传输的准 确性和安全性。比如在切削机械中应用机电一体化数控技术能够实现实时监测机械运行时各项数据的目的。切削功率 的计算公式为 $p=P \times N \times K$, 其中公式中的 $P$ 为主电机的实际功率, $N$ 为主传动系数的总功率数值, $K$ 为进给系统的功率 系数。在传感器获取这 3 项数值之后, 将之传输到指令中心, 经过计算后便可得到实际的切削功率。同时, 机械控制 系统可以及时获取加工工件精度、机床状态等数据信息，从而对整个机械设备进行有效的监控。

\section{2 应用在监控系统部分}

机械生产加工过程中很容易出现突发状况, 其中加工原材料供应不足和设备故障时最为常见的两种现象。及其生 产加工设备需要在遇到这些突发情况时及时停止工作, 避免对设备或者员工产生伤害。此时, 监控系统的价值就可以 体现出来。利用监控系统能够对机械生产过程中各个系统的情况进行实时监测, 同时在机电一体化数控技术中应用监 控系统可以实现故障诊断和自动报警, 能够在设备发生故障或者其他异常情况时第一时间做出判断, 将故障位置确定 并且给出初步判断结果, 向工作人员发出提醒。为了有效达到这一效果, 需要在监控系统中将各个记下加工生产设施 本体结构以及组成部分的实际参数输入到系统当中, 监控系统可以监测运转中的机械设备, 并且同步对比正常运行下 的各项参数, 如果发生较大的数值差异会报警或者下达暂停指令。

\section{3 机电一体化数控技术应用优势}

\section{1 完善工业系统}

经过多年发展和研究, 我国已经积累了大量的机械制造相关工作经验, 不过和发达技术相比差距仍然较大。通过 完善和优化机电一体化技术有助于我国工业化体系的进步，能够将我国工业化体系中的不足及时发现并且改进，更好 地推动整个机械加工行业发展。

\section{2 控制加工精度}

在机械加工中利用传感器能够及时获取和调整设备运行的各类参数, 能够通过分析实际运行数据来将现存问题及 时挖掘出来, 从而有针对性地进行整改, 有助于推动机械加工的发展。反馈系统可以过滤掉干扰信息, 对比各类参数, 加上合理设计系统能够消除无用信息, 实现控制精准度提升的效果。

\section{3 提高加工效率}

在机械加工中应用机电一体化数控该系统可以将控制的精度提升, 进而将机械加工的效率和加工系统对接工作效 率提升。此外, 在具体应用数控技术过程中能够将加工精确度提升实现返工概率降低的效果, 进而缩短机械加工时间, 高效完成机械加工作业。

\section{4 机电一体化数控技术应用策略}

\section{1 自动化数控系统的应用}

数控系统在机电一体化工程中有着非常重要的地位, 数控技术能够标准化完成装配、焊接、拼接等工作内容, 充 分发挥工业机器人的作用。同时, 在恶劣的环境下, 自动化控制技术能够完成人工难以完成的工作, 比如利用机器人 手臂运输、抓取零件, 避免恶劣环境影响操作人员的身心健康。在实际应用自动化数控系统时需要首先做好控制单元 的构建, 从而实现检测、执行动作同步完成。实际工作中需要借助计算机等终端设备进行控制单元的操作, 比如结合 利用控制单元和计算机系统, 实现中枢神经的构建, 并且用计算机规范化指导机器人完成指定的任务。如果在工作中 机器人出现了故障, 传感器可以将故障信息及时回传反馈给终端设备计算机等控制单元, 并且发出警报, 工作人员及 时将出现的故障解决。

\section{2 自动调整机械控制模块}

机械控制模块主要是执行实施调整角度的工作, 模块控制的精准度直接影响机械加工中切材与圆盘切刀相对角度 
精度。角度调整模块、联轴器、伺服电机共同组成了角度实时调整机械控制模块, 可以将调整期间轴肩与圆盘切刀的 冲击降低, 通常调整原件材料为柔性材质的橡胶, 选用这种材质可以达到材料处理后的高端面质量优化的效果。

\section{3 机电一体化技术与机械制造的结合}

在应用机电一体化技术过程中可以充分发挥智能化数字机床的作用, 对刀具进行合理选择, 保证机床加工零件能 够满足实际需要。在具体应用中需要重点做好如下工作：第一，及时切换产品, 精准地控制机械制造的参数。第二, 为解决常规单件加工速度慢的问题可以采用一次多工件多工序加工的方式, 并且比较加工后的成品, 提高加工质量。 第三, 稳定地使用机电一体化加工技术。第四, 为了将刀具安装时间缩短可以建设标准化的机械制造模块, 做好标准 化制造模式的区分。

\section{4 优化机械设计制造集成化应用}

在机械制造中应用集成技术可以对企业当前的整体生产状况有清晰的了解, 并且有助于改进技术的应用。比如当 前电梯机械设计制造中应用集成化制造技术效果良好, 能够实现电梯制造质量的提升。加强应用集成化机械设计制造 技术能够提升机械设计制造中每项管理活动效果, 有助于对各种资源和材料进行集中管理和调整, 实现资源的高效利 用, 将设计制造功能性充分彰显出来。当前信息技术、多媒体技术、计算机图形技术等都是常见的自动化数控技术虚 拟化技术。CPU 及可编程集成电路板是当前集成化技术应用主要依靠的设备, 为了保证集成化技术的高效应用以及机械 加工系统稳定地运行就要合理选择集成系统、软硬件云心速度、集成电路等。目前机械设计制造领域的基础技术之一 就是集成技术, 通过利用集成化技术能够将产品生产制造的成本降低。比如在各类现代化设备制造中应用集成化技术 能够将生产效率提高, 能够提出具体优化和解决问题的方案, 有助于将产品开发周期缩短, 有助于合理设置辅助模拟 自动参数, 实现整体数据可视化管理。

\section{5 结语}

将机电一体化数控技术应用于机械制造行业可以将机械制造加工水平不断提高, 节省人员, 减轻一线员工的工作 量, 实现智能化控制切割, 自动监测设备故障, 有助于提高机械加工的安全性。当前数控技术在应用中能够发挥优良 的效果, 是值得未来进一步推广研究的一项技术。

\section{[参考文献]}

[1]滑雪燕. 机电一体化数控技术在机械制造中的应用 [J]. 时代农机, 2017, 44 (4) : 25-26.

[2]张祝强. 机电一体化数控技术在机械加工中的应用 [J].中国设备工程, 2017 (6) : 117-118.

[3]王丽巍. 机电一体化数控技术在机械制造中的实践研究 [J]. 科技经济导刊, 2017 (4) : 53 .

[4] 段斌. 机电一体化数控技术的发展趋势研究 [J].中国高新区, 2017 (2): 90 .

作者简介: 王卫平 (1969-) 男, 江西江特电机有限公司, 研发中心, 总工程师/专家, 高级工程师, 专业: 机电一体 化, 电机研发设计。 